\title{
Pharmaceutical Quality of Nine Generic Orlistat Products Compared with Xenical ${ }^{\circledR}$
}

\author{
Peter W. Taylor ${ }^{a}$ Isabelle Arnet ${ }^{b}$ Anton Fischer ${ }^{c}$ lain N. Simpson ${ }^{d}$ \\ ${ }^{a}$ London School of Pharmacy, London, UK \\ ${ }^{\mathrm{b}}$ Pharmaceutical Care Research Group, University of Basel, \\ ${ }^{\mathrm{C}}$ F. Hoffmann - La Roche Ltd. Basel, Switzerland \\ ${ }^{\mathrm{d}}$ The Micron Group, Chatteris, UK
}

\section{Keywords}

Orlistat $\cdot$ Xenical $\cdot$ Pharmaceutical quality $\cdot$ Generics

\section{Summary}

Objective: To compare the pharmaceutical quality of Xenical (chemically produced orlistat) with nine generic products, each produced by fermentation processes. Methods: Xenical $120 \mathrm{mg}$ capsules (Roche, Basel, Switzerland) were used as reference material. Generic products were from India, Malaysia, Argentina, Philippines, Uruguay, and Taiwan. Colour, melting temperature, crystalline form, particle size, capsule fill mass, active pharmaceutical ingredient content, amount of impurities, and dissolution were compared. Standard physical and chemical laboratory tests were those developed by Roche for Xenical. Results: All nine generic products failed the Xenical specifications in four or more tests, and two generic products failed in seven tests. A failure common to all generic products was the amount of impurities present, mostly due to different by-products, including side-chain homologues not present in Xenical. Some impurities were unidentified. Two generic products tested failed the dissolution test, one product formed a capsule-shaped agglomerate on storage and resulted in poor $(\leq 15 \%)$ dissolution. Six generic products were powder formulations. Conclusions: All tested generic orlistat products were pharmaceutically inferior to Xenical. The high levels of impurities in generic orlistat products are a major safety and tolerability concern.

\section{Introduction}

Orlistat (N-formyl-L-leucine (S)-1-[[(2S,3S)-3-hexyl-4-oxooxetan-2-yl]methyl]dodecyl ester) (fig. 1) is a potent irreversible inhibitor of gastrointestinal lipases that hydrolyse triacylglycerols in the gastrointestinal tract to free fatty acids and monoacylglycerols $[1,2]$. This inhibition prevents absorption of up to one third of all dietary fat, [3] and therefore can promote weight loss, maintain lost weight, and prevent weight regain in obese patients [4]. The relationship between orlistat daily dose and fecal fat excretion is determined by gastrointestinal lipase inhibition [3]. The dose-response curve demonstrates a steep portion for doses of up to approximately $400 \mathrm{mg}$ daily. The orlistat daily dose that produces $50 \%$ of the maximum effect is approximately $100 \mathrm{mg} /$ day.

Orlistat is patented and chemically synthesised using stereospecific methods which result in $\geq 99.5 \%$ pure product. It is manufactured and marketed as $120 \mathrm{mg}$ capsules under the brand name Xenical by F. Hoffmann-La Roche Ltd (Roche, Basel, Switzerland) since its launch in 1998. Xenical has proven to be an invaluable drug in the long-term treatment of obese and overweight patients. As of July 2009, more than 35 million patients had received Xenical. During research and development, Roche also evaluated a less expensive fermentation process to produce orlistat using the microorganism Streptomyces toxytricini. However, chemical analyses revealed a considerable range of impurities in the final product, and this process was abandoned.

Orlistat is polymorphic, existing in distinct crystalline forms A and B. Xenical is polymorph form B. Orlistat is highly lipophilic and practically insoluble in water, having no $\mathrm{pK}_{\mathrm{a}}$ value within physiological $\mathrm{pH}$. Orlistat powder is 'sticky', which has consequences for manufacture and dispensing into capsules. Roche has overcome these difficulties in Xenical capsule production by using a patented granular 'pellets' for-

\begin{tabular}{ll}
\hline KARGER & @ 2010 S. Karger GmbH, Freiburg \\
Fax +497614520714 & Accessible online at: \\
Information@Karger.de & www.karger.com/ofa \\
www.karger.com &
\end{tabular}


mulation that includes a disintegrant [5]. Stability tests of Xenical carried out by Roche revealed a slow decline in dissolution rates over the 3-year shelf life at a storage temperature of $25^{\circ} \mathrm{C}$ and relative humidity of $60 \%$.

Although the Xenical patent has expired in 2009, generic orlistat had been manufactured and available for purchase for several years in those countries whose national laws do not support patent protection.

The current study compares the pharmaceutical quality of Xenical with nine generic products. All products were assessed against Roche standard operating procedures (SOPs) that describe the qualitative and quantitative pharmaceutical tests for physical and chemical purity of orlistat from both chemical and fermentative synthesis processes. The analysis processes for chemically synthesised orlistat were accepted by regulatory authorities as part of the Xenical registration documentation. Values of reference were the official Roche specifications for product testing at release or at shelf-life, where appropriate.

\section{Material and Methods}

\section{Generic Products}

Samples of nine generic orlistat products, all $120 \mathrm{mg}$ capsule preparations, were purchased for testing (table 1)

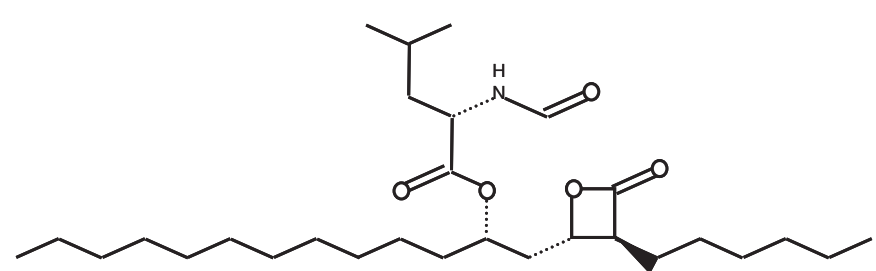

Fig. 1. Chemical structure of orlistat.
Pharmaceutical Quality Tests

The tests were colour of capsule contents, identification of the active pharmaceutical ingredient (API) and the crystalline form, melting temperature, particle size, dissolution, fill mass of capsules, content of orlistat and of impurities.

\section{Colour of Capsule Contents}

Colour was assessed visually according to the standards described in the 'Munsell Book of Color' [6].

\section{Identification of API and Crystalline Form}

Infrared spectroscopy was used with a suspension of $5 \mathrm{mg}$ capsule content in Nujol/sodium chloride plates. The spectrum was recorded in the range of 4,000-650/cm using a Nicolet 20 SXB spectrophotometer and compared to the reference spectra of the polymorphic forms A and B of orlistat.

\section{Melting Temperature}

Melting temperature of the capsule content was determined using a Mettler DSC-821e DSC module equipped with a Mettler TSO801RO sample robot and STARe, TA-8000 evaluation and control system (Mettler Toledo, Greifensee, Switzerland). Determinations were made using between 6.0 and $13.0 \mathrm{mg}$ of capsule content in a $40 \mu \mathrm{l}$ sealed aluminium crucible under a nitrogen atmosphere, across the temperature range $25-60{ }^{\circ} \mathrm{C}$, and using a ramp rate of $1{ }^{\circ} \mathrm{C} / \mathrm{min}$. Polymorphic form $\mathrm{B}$ has a melting point of $43.6^{\circ} \mathrm{C}$.

\section{Particle Size}

Particle size analysis was performed by laser diffraction, using a MasterSizer 2000, (Malvern Instruments, Worcestershire, UK) fitted with a Scirocco 2000 dry powder feeder (Malvern Instruments) operated at $25{ }^{\circ} \mathrm{C}$ with a measuring time of $15 \mathrm{~s}$, a feed rate of approximately $60 \%$, and obscuration limits set between 0.1 and $6 \%$. The entire content of one capsule was used per determination; 3,6 , or 9 separate determinations were made, and an average was taken. Xenical pellets show a mean particle size distribution of D50 $=1,100 \mu \mathrm{m}$ and D $90=1,500 \mu \mathrm{m}$. The D50 and D90 represent the median or 50th percentile and the 90th percentile of the particle size distribution, respectively, as measured by volume.

\section{Dissolution}

Dissolution testing was performed using the $\mathrm{Ph}$. Eur. rotating paddle apparatus in a dissolution medium that was developed especially for orlistat

Table 1. Details of Xenical and generic orlistat products tested

\begin{tabular}{|c|c|c|c|c|c|c|c|}
\hline Product & Manufacturer & Country & Batch & Manufacture date & Expiry date & $\begin{array}{l}\text { Shelf-life, } \\
\text { months }\end{array}$ & Test date \\
\hline Xenical & Roche Pharmaceuticals & Switzerland & B2637 & July 2006 & July 2009 & 36 & August 2006 \\
\hline Cobese & Ranbaxy & India & $\begin{array}{l}1941737 \\
1792387 \\
1853969\end{array}$ & $\begin{array}{l}\text { Aug. } 2008 \\
\text { July } 2007 \\
\text { January } 2008\end{array}$ & $\begin{array}{l}\text { June } 2010 \\
\text { May } 2009 \\
\text { December } 2009\end{array}$ & $\begin{array}{l}24 \\
22 \\
24\end{array}$ & December 2008 \\
\hline Cuvarlix & Pharmaniaga & Malaysia & 8L184 & September 2008 & September 2010 & 24 & February 2009 \\
\hline Fingras & Phoenix & Argentina & 15026 & (1) & (1) & unknown & December 2006 \\
\hline Lesofat & InnoGen Pharma Group & Philippines & 7E149B & May 2007 & May 2009 & 24 & November 2007 \\
\hline Obelit & Intas Pharma & India & H5018W & (1) & (1) & unknown & December 2005 \\
\hline Xeniplus & Elea & Argentina & L8114 & (1) & (1) & unknown & December 2006 \\
\hline Xiluet & Servimedic & Uruguay & 01 & (1) & February 2008 & unknown & April 2007 \\
\hline Xinplex & Craveri & Argentina & 9661 & (1) & (1) & unknown & December 2006 \\
\hline Zerocal & $\begin{array}{l}\text { Pharmosa/Weidar Chemical } \\
\text { and Pharmaceutical Co. Ltd }\end{array}$ & Taiwan & 735002 & February 2007 & February 2009 & 24 & May 2007 \\
\hline
\end{tabular}

(1) = Not stated on packaging. 
substance due to its hydrophobicity. The dissolution medium comprised an aqueous solution containing $3 \%$ of sodium lauryl sulphate, $0.5 \%$ of sodium chloride, adjusted to $\mathrm{pH} 6.0$ with phosphoric acid; sink conditions were achieved because the solubility of orlistat in this medium is approximately $0.3 \mathrm{~g}$ in $100 \mathrm{ml}$. The rate of dissolution was determined individually for six capsules of each generic product, in a vessel with a paddle stirrer at $75 \mathrm{rpm}$ and containing $900 \mathrm{ml}$ of medium. Aliquots of $10 \mathrm{ml}$ were removed after 15, 30, 45 and $60 \mathrm{~min}$. These aliquots were filtered through a $1 \mu \mathrm{m}$ Acrodisc glass fibre filter or $0.2 \mu \mathrm{m}$ Acrodisc filter (Pall Medical, Milan, Italy) and cooled to $20^{\circ} \mathrm{C}$, and $20 \mu \mathrm{l}$ samples of the resultant clear solution were assayed using high performance liquid chromatography (HPLC) and spectrophotometry. The specification for Xenical dissolution rate at shelflife was a Q-value of $65 \%$ after $45 \mathrm{~min}$ according to $\mathrm{Ph}$. Eur.

\section{Fill Mass of Capsules}

Twenty capsules were emptied, their contents mixed together weighed and the average weight calculated. Xenical specification for fill mass was 228.2-252.2 mg per capsule.

\section{Content of Orlistat and of Impurities}

The content of orlistat and impurities was determined using HPLC Twenty capsules were emptied and their contents mixed together, from which $100 \mathrm{mg}$ was dissolved in $70 \mathrm{ml}$ mobile phase, agitated by ultrasound for $1 \mathrm{~min}$, then made up to $100 \mathrm{ml}$ before being agitated for a further $15 \mathrm{~min}$. A portion of the resulting solution was filtered $(0.45 \mu \mathrm{m}$ pore size, Millex HV; Millipore, Volketswil, Switzerland) and stored for up to $48 \mathrm{~h}$ at $4{ }^{\circ} \mathrm{C}$. Samples $(20 \mu \mathrm{l})$ were then injected onto the HPLC analytical column $(150 \mathrm{~mm} \times 3.9 \mathrm{~mm}$ Nova Pak C18, $4 \mu \mathrm{m}$; Waters, Baden-Dättwil, Switzerland), and eluates monitored at $195 \mathrm{~nm}$ (Hewlett Packard G1314A variable wavelength detector). The mobile phase comprised acetonitrile $(860 \mathrm{ml})$, water $(140 \mathrm{ml})$ and $5 \%$ phosphoric acid $(1 \mathrm{ml})$. Mobile phases were degassed (Hewlett Packard G1322A vacuum degasser) and pumped (Hewlett Packard G1311A quat pump) at $1.0 \mathrm{ml} / \mathrm{min}$.

The specification for content of API was $114.0-126.0 \mathrm{mg}(95-105 \%)$ orlistat per capsule of Xenical. The specification for the limit for all impurities in Xenical was $0.5 \%$ and $2.5 \%$ at release and at shelf-life, respectively. The shelf-life limits were between 0.2 and $1.2 \%$ for five specific degradation products. Each other characterised impurity, which included precursors, stereoisomers, side-chain homologues, amino acid analogues or other degradation products, was limited at $0.3 \%$. The limits were $0.5 \%$ for all unidentified impurities at shelf-life.
All pharmaceutical quality tests were conducted in GMP-regulated Quality Control and Development Laboratories of F. Hoffman LaRoche, in Basel, by certified personnel.

\section{Results}

Five generic products did not fully detail dates of manufacture and/or expiry (table 1). The age of product when tested was unknown for Fingras, Obelit, Xeniplus, and Xinplex. Xiluet was tested within 10 months of expiry. The remaining generic products were tested between 3 and 6 months of manufacture. Where stated, the maximum shelf life claimed for generic products was 2 years, i.e. 1 year less than Xenical.

\section{Colour, Appearance, Crystalline Form, and Melting Temperature}

All generic products were white or off-white in colour, similar to Xenical (table 2). Lesofat, Xiluet, and Xinplex were pelleted or granular formulations resembling Xenical; however, five of the remaining generic products were powders and the contents of the Cuvarlix capsules remained in a single lump (fig. 2). Six of the generic products consisted solely of polymorphic form A and three products (Xiluet, Fingras, and Xinplex) were mixtures of form A and B. Melting temperatures were determined for six generic products, and all were similar to Xenical.

\section{Particle Size Distribution}

Xenical exhibited a narrow range particle size distribution with a median (D50) of 1,100 $\mu \mathrm{m}$ (fig. 3). Cobese, a typical powder formulation, exhibited a wider range of particle sizes with bimodal values of 100 and $900 \mu \mathrm{m}$. Particle size distributions of the granular formulations Xiluet, Xinplex, and Lesofat were similar to that of Xenical (fig. 4), with a median size

Table 2. Physical characteristics of Xenical and generic orlistat products, following removal from capsules

\begin{tabular}{|c|c|c|c|c|c|c|}
\hline \multirow[t]{2}{*}{ Drug product } & \multicolumn{2}{|l|}{ Description } & \multirow{2}{*}{$\begin{array}{l}\text { Melting } \\
\text { temperature }{ }^{\mathrm{a}},{ }^{\circ} \mathrm{C}\end{array}$} & \multirow{2}{*}{$\begin{array}{l}\text { Polymorphic } \\
\text { crystalline form }\end{array}$} & \multirow{2}{*}{$\begin{array}{l}\text { Average fill } \\
\text { mass }^{\mathrm{b}}, \mathrm{mg}\end{array}$} & \multirow{2}{*}{$\begin{array}{l}\text { Average orlistat } \\
\text { content, } \mathrm{mg}\end{array}$} \\
\hline & colour & appearance & & & & \\
\hline Xenical & white / off-white & pellets & 43.6 & $\mathrm{~B}$ & 240.6 & 118.3 \\
\hline Cobese & almost white & powder & 43.1 & A & 231.2 & 113.7 \\
\hline Cuvarlix & off-white & highly agglomerated powder & 44.8 & A & 243.8 & 120.1 \\
\hline Fingras & off-white & powder & 42.2 & $\mathrm{~B}$ and $\mathrm{A}$ & 248.9 & 116.2 \\
\hline Obelit & off-white & powder & NT & A & 260.6 & 114.6 \\
\hline Xeniplus & off-white & powder & 42.2 & A & 252.3 & 117.8 \\
\hline Xiluet & off-white & spheroids & NT & $\mathrm{A}$ and $\mathrm{B}$ & 332.1 & 116.9 \\
\hline Xinplex & off-white & irregular balls & 41.1 & B (some A) & 244.7 & 114.1 \\
\hline Zerocal & white & powder & NT & A & 184.4 & 126.4 \\
\hline
\end{tabular}

NT $=$ Not tested

a Specification $42-44^{\circ} \mathrm{C}$.

${ }^{\mathrm{b}}$ Specification 228.2-252.2 $\mathrm{mg}$.

'Specification 114.0-126.0 mg. 
Fig. 2. Physical appearance of capsules and contents of Xenical and three generic products.

Fig. 3. Comparison of particle size distribution for Xenical and Cobese.

Fig. 4. Particle size distribution of Xenical and generic orlistat products.

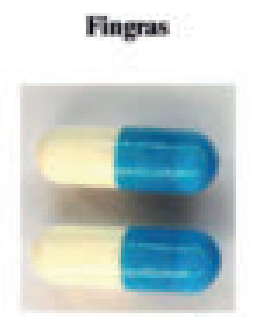

Cuvarlix

Lesofat

Xenicale
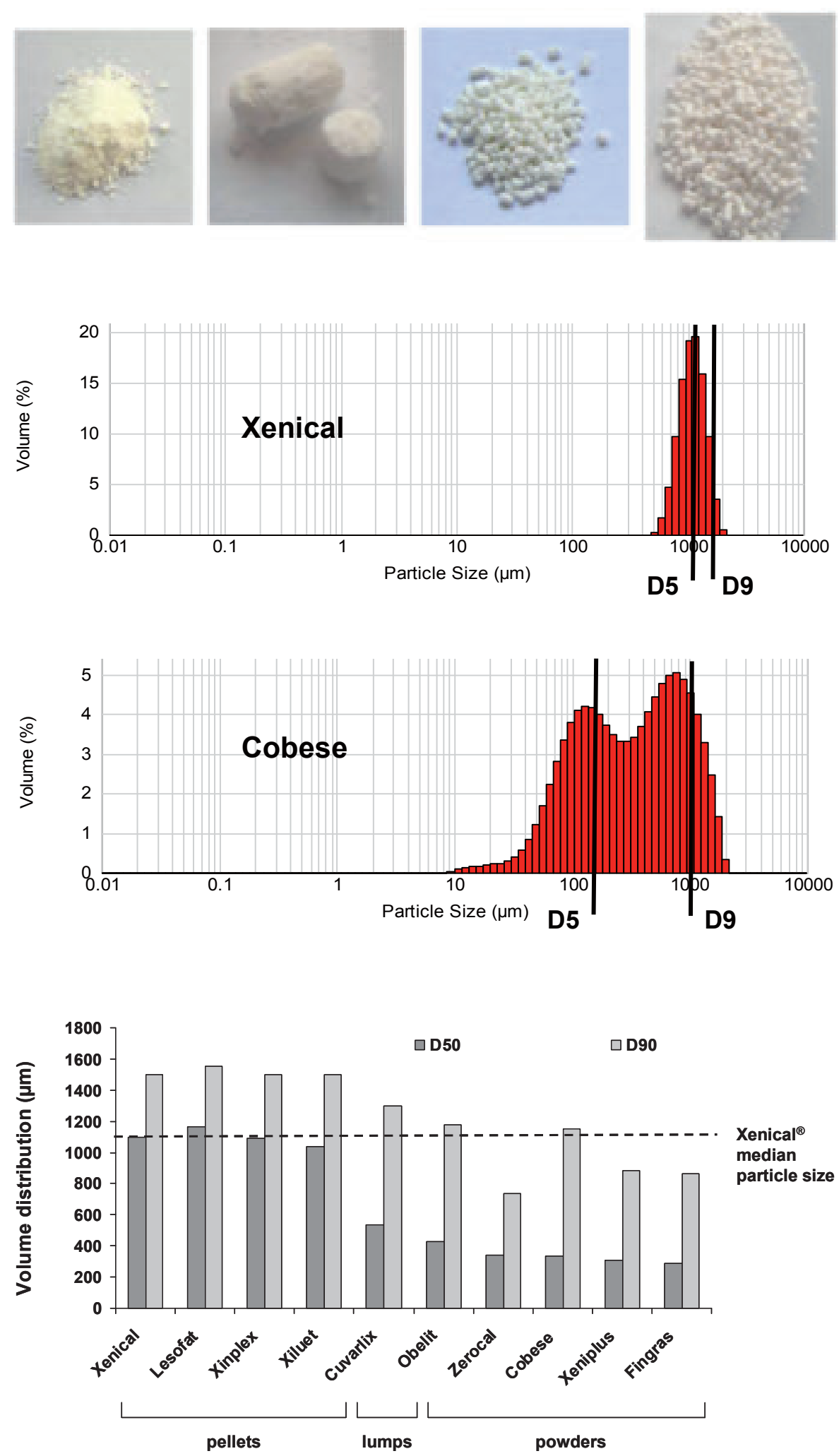

Obes Facts 2010:3:231-237 
Fig. 5. Summary of impurities (\%) in Xenical and generic orlistat products: A Total organic impurity content. B Total side chain homologues. C Unidentified impurities.

Fig. 6. Dissolution rate (\% at $45 \mathrm{~min}$ ) for Xenical and generic products.
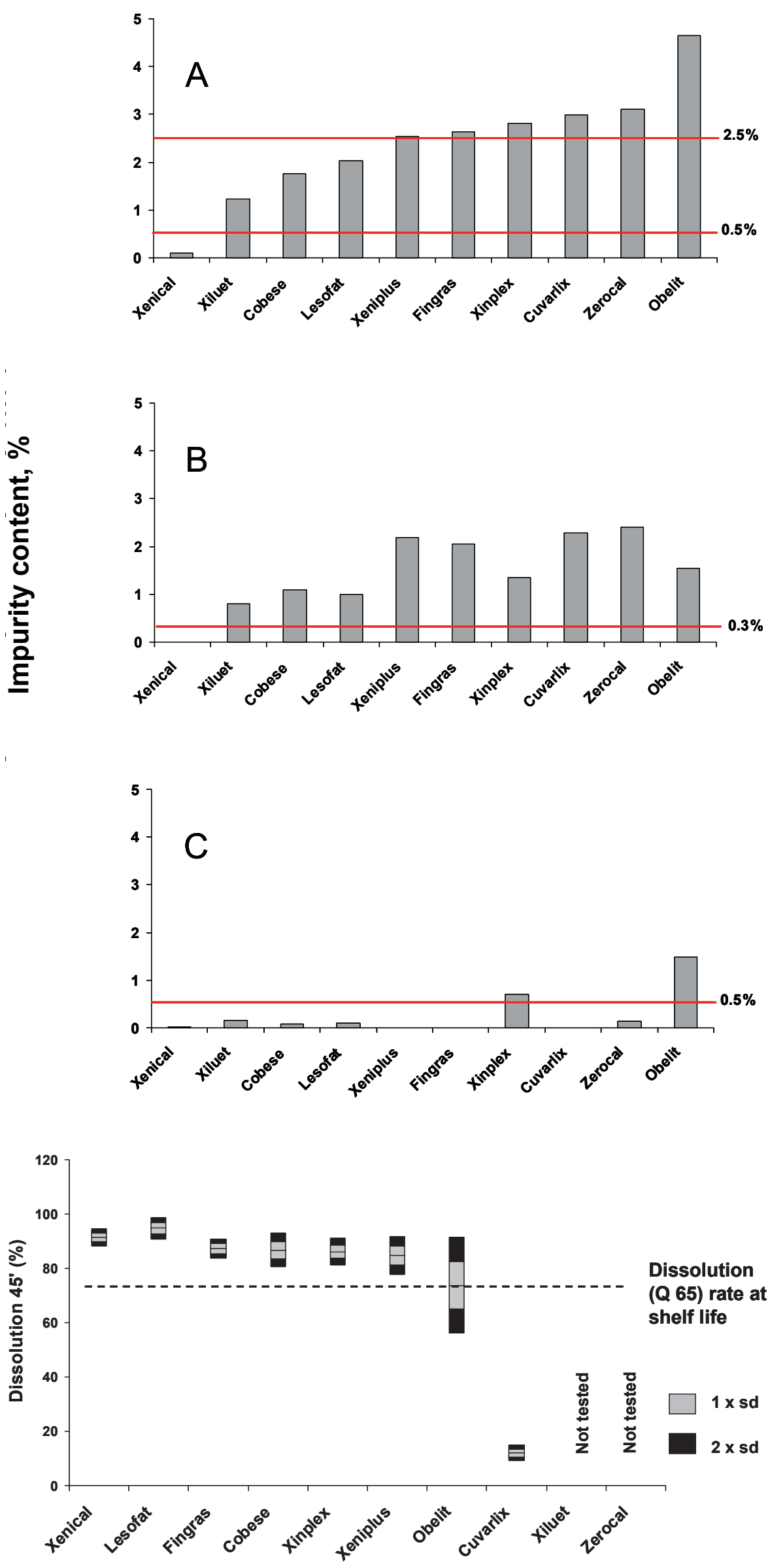
of 1,100 $\mu \mathrm{m}$. Particle sizes of five 'powder' form generics (Fingras, Xeniplus, Cobese, Zerocal, and Obelit) were generally much smaller, with median particle size of $\leq 500 \mu \mathrm{m}$.

\section{Fill Mass and Orlistat Content of Capsules}

The average fill mass of five of the nine generic products fell within the Roche specification of 228.2-252.2 mg per capsule (table 2). Three generic products (Xeniplus, Obelit, and Xiluet) exceeded the upper limit by up to $31.7 \%$ (Xiluet $332.1 \mathrm{mg}$ ), whereas Zerocal fell below the lower limit by $19 \%$ (184.4 mg). The average orlistat content requirement of $114.0-126.0 \mathrm{mg}$ per capsule was met by six generic products, whilst Zerocal (126.4 mg) exceeded specification.

\section{Impurities}

All generic products failed to meet the Roche specification for the total of all impurities of $0.5 \%$ at release, and five generic products failed to meet the specification of $2.5 \%$ at shelf-life (range 1.2-4.6\%), (fig. 5A). The impurities observed in the generic products were due to different chemicals, including side-chain homologues and amino acid analogues not present in Xenical.

All nine generic products contained side-chain homologues (range $0.8-2.4 \%$, fig. 5B). Three generic products (Cuvarlix, Xeniplus, and Xinplex) each contained excessive amounts of at least four individual side-chain homologues (data not shown).

Six generic products contained higher levels of unidentified impurities than Xenical. Two generic products, Obelit $(1.5 \%)$ and Xinplex $(0.71 \%)$, exceeded specification of $0.5 \%$ (fig. 5C).

\section{Dissolution Rate}

Five generic products fully satisfied the $65 \%$ Q-value shelflife specification for dissolution rates (fig. 6). Obelit exhibited marked variation in dissolution rates with many values falling below the acceptable dissolution rate; Cuvarlix exhibited very poor dissolution $(\leq 15 \%)$.

\section{Number of Violations of Roche Specifications}

All generic products failed to match Xenical in four or more tests, and two generic products failed in seven tests (table 3 ). A failure common to all generic products was the amount of impurities present, some of which were unidentified. Two generic products tested failed the dissolution test.

\section{Discussion}

Orlistat is polymorphic, existing in two distinct crystalline forms with similar physical properties in terms of solubility, dissolution rate of the pure substance, and hygroscopicity. This explains our findings of similar melting temperatures among the generic products tested compared to Xenical.

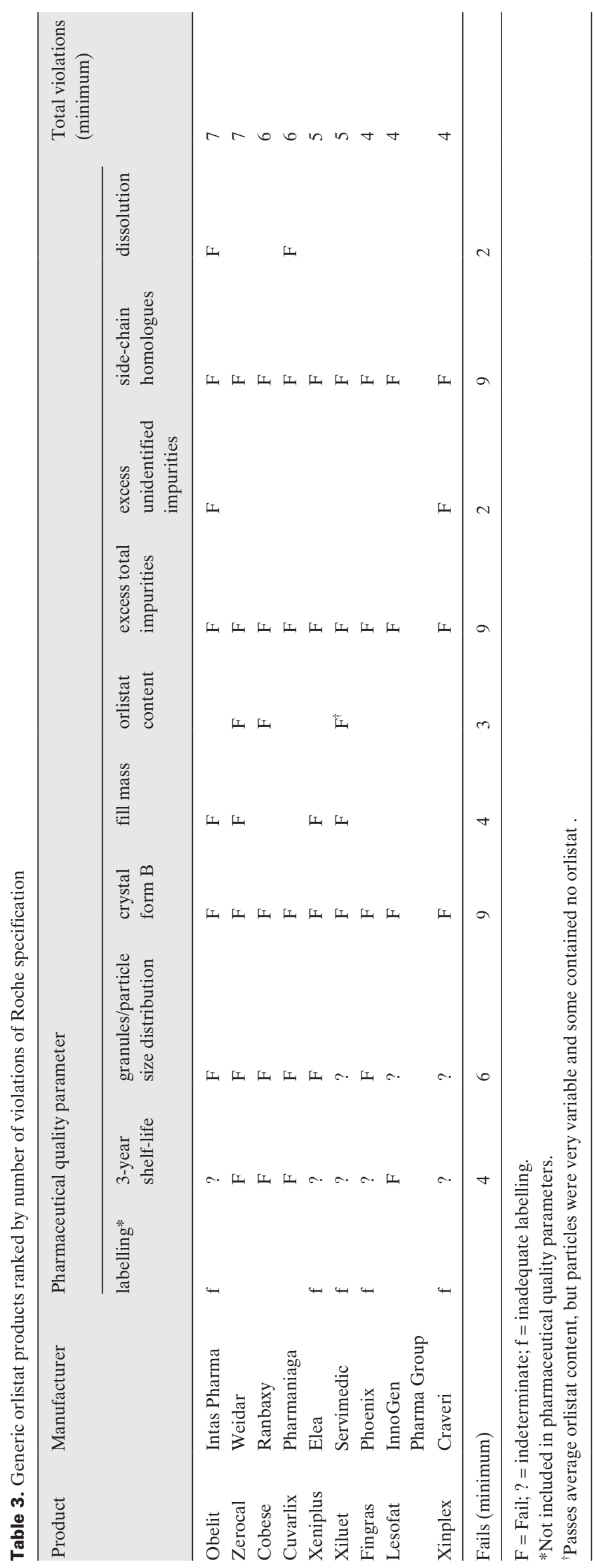

Obes Facts 2010;3:231-237 
Previous studies have found some generic products to be less uniform in terms of unit fill mass and amount of active ingredient and to contain more impurities than the innovator product [7-11]. We compared the pharmaceutical quality of synthetically produced orlistat (Xenical) with nine generic products. The wide spectrum and identity of impurities was consistent with these products being produced via fermentation processes. We could identify two areas of concern: dissolution rate and impurity content, with corresponding potential consequences on dose delivery and toxicological issues, respectively. Both may also have an impact on efficacy and safety, respectively. We did not include the wholesale or retail costs of products although this can be a major argument for the development and usage of generic products.

Stability tests of Xenical carried out by Roche revealed a slow decline in dissolution rates over a 3 -year period at a storage temperature of $25{ }^{\circ} \mathrm{C}$ and $60 \%$ relative humidity. Although successful dissolution in vitro does not guarantee equivalent dissolution in vivo and does not always reflect in vivo availability, such tests are required for registration to demonstrate batch-to-batch consistency during manufacture. The dose-response of orlistat exhibits considerable variability among individuals [3], which was attributed to how well orlistat mixed with the fat content of a meal [12]. This step might be influenced by the dissolution of the product. Suggestively, in a clinical comparison of orlistat formulations, a granulated formulation tended towards superior efficacy over a powdered formulation in increasing dietary fat excretion [13]. In this context, the very low dissolution rate shown by Cuvarlix and the large variation in dissolution rates observed with $\mathrm{Ob}$ elit are causes for clinical concern as they indicate that little orlistat might be available and, hence, that the products would be expected to have markedly reduced efficacy. Such clinical efficacy concerns with generic products can only be addressed by controlled clinical studies, designed to meet the same endpoints as in the original study, that is faecal fat excretion and/ or weight loss over time. The cost of conducting such studies should be borne by the generic manufacturers, rather than the original patent holder. If a generic product achieves the same weight loss for a much lower cost, then it could be argued that the pharmaceutical differences, in terms of efficacy, are not important.

For the tested generic products, the spectrum and amount of impurities reflect the complex biological processes inherent in fermentation and the increased difficulties in extracting pure product. A more serious finding, however, was the relatively large amount of unidentified organic material in Obelit and Xinplex, which raises toxicological questions for these products. As orlistat formulations may be given to patients over long periods of time, possibly for the rest of their lives, the issue of toxicological effects of impurities may be serious due to their possible accumulation in these patients. As with the requirement for efficacy studies, the onus should be on generic manufacturers to provide clinical evidence that their products, particularly when less pure than the patented product, are not associated with additional side-effects.

\section{Conclusions}

The pharmaceutical quality of nine generic products was compared with Xenical. The wide spectrum of impurities indicates that the generic products were produced using fermentative processes. On the basis of the poor dissolution rates and the wide spectrum of impurities observed, we must question whether the nine generic products tested can be said to represent pure, safe and consistent alternatives to Xenical.

\section{Disclosure}

PWT, IA and INS have provided consultancy services to F. Hoffman La-Roche Ltd. AF is an employee of F. Hoffman La-Roche Ltd.

\section{References}

1 Hadvary P, Lengsfeld H, Wolfer H: Inhibition of pancreatic lipase in vitro by the covalent inhibitor tetrahydrolipstatin. Biochem J 1988;256:357-361.

2 Borgström B: Mode of action of tetrahydrolipstatin: a derivative of the naturally occurring lipase inhibitor lipstatin. Biochim Biophys Acta 1988;962:308-316.

3 Lucas KH, Kaplan-Machlis B: Orlistat - a novel weight loss therapy. Ann Pharmacother 2001;35: 314-328.

4 Zhi J, Melia AT, Guerciolini R, Chung J, Kinberg J, Hauptman JB, Patel IHL: Retrospective populationbased analysis of the dose-response (fecal fat excretion) relationship of orlistat in normal and obese volunteers. Clin Pharmacol Ther 1994;56:82-85.
5 United States Patent and Trademark Office. Patent 4.598.089 Extension, June 18, 2004. www.uspto.gov/ web/offices/pac/dapp/opla/term/certs/4598089.pdf.

6 Munsell Book of Color. New Windsor, GretagMacbeth, 1996 .

7 Nightingale CH: A survey of the quality of generic clarithromycin products manufactured in Slovenia and Israel. Adv Ther 2000;17:167-178.

$\checkmark 8$ Lambert PA, Conway BR: Pharmaceutical quality of ceftriaxone generic drug products compared with Rocephin. J Chemother 2003;15:357-368.

9 Nightingale $\mathrm{CH}$ : A survey of the quality of generic clarithromycin products from 18 countries. Clin Drug Investig 2005;25:135-152.

10 Taylor PW, Keenan MHJ: Pharmaceutical quality of generic isotretinoin products compared with Roaccutane. Curr Med Res Opin 2006;22:603-615.
11 Smith JC, Tarocco G, Merazzi F, Salzmann U: Are generic formulations of carvedilol of inferior pharmaceutical quality compared with the branded formulations? Curr Med Res Opin 2006;22:709-720.

12 Carriere F, Renou C, Ransac S, Lopez V, De Caro J, Ferrato F, De Caro A, Fleury A, SanwaldDucray P, Lengsfeld H, Beglinger C, Hadvary P, Verger R, Laugier R: Inhibition of gastrointestinal lipolysis by orlistat during digestion of test meals in healthy volunteers. Am J Physiol Gastrointest Liver Physiol 2001;281:G16-G28.

13. Hartmann D, Güzelhan C, Crijns HJMJ, Peeters PAM, Persson P, Jonkman JHG: Comparison of galenic formulations of orlistat (tetrahydrolipstatin). A pharmacological approach. Drug Invest 1993;5:44-50 